\title{
DESIGN HINTS FOR SMART AGENTS AS TEACHERS IN VIRTUAL LEARNING SPACES
}

\author{
Thomas Keller and Elke Brucker-Kley \\ ZHAW School of Management and Law, Winterthur, Switzerland
}

\begin{abstract}
Immersive virtual learning environments can be used to teach a wide range of competences. Usually the learners are alone in such learning worlds. For help, they then necessarily turn to their class teacher. An alternative is the use of a smart agent as an aid. This approach is known as intelligent tutorship. The difference, however, lies in the immersive nature of the learning environment. This work is based on a literature review to identify requirements for smart agents in the context of a virtual classroom. In addition to multimodal conversation skills and pedagogical competences, the role as a central point of interaction and mentor/tutor is also part of this. The outer appearance (i.e., embodiment) should have a visible shape and be animated. The second part of the work deals with the practical conception of a smart agent. By means of a survey, the preferred visualization of a smart agent for a specific target group was identified. This showed that smart agents with human appearance were preferred.
\end{abstract}

\section{KEYWORDS}

Virtual Reality, Classroom, Interaction, Visualization, smart agent, Intelligent Tutor System

\section{INTRODUCTION}

Starting point of the present work are three developed immersive learning concepts based on virtual reality ((Keller et al., 2019), (Keller, Hebeisen, et al., 2018), (Keller, Glauser, et al., 2018)). The target group are learners aged 12 to 14 years, who are supposed to solve tasks on different topics in virtual space. Currently, concepts on probability, microplastics and mathematics ${ }^{1}$ are available. The object of investigation is the integration of a smart agent into these concepts. This should be available to the learner as an advisor during the course of a learning unit and should be used if the learner is not making any progress with a task.

From the secondary research carried out ((Jawahar, 2015), (A. L. Baylor, 2009), (Kim \& Baylor, 2016), (Baylor \& Kim, 2005), (Y. Kim et al., 2006), (Aleven \& Koedinger, 2000), (Baylor, 2011), (K. Kim et al., 2018), (Fasihfar \& Rokhsati, 2017)) it could be determined that software agents in the pedagogical field are to be found primarily in connection with eLearning applications. There, the intelligent agents act as tutors, mentors or teachers who teach the learners different learning contents didactically. The appearance, facial expressions, gestures and behaviour of the agent have an influence on the perception, acceptance and performance of the learners.

The latter in particular can be negatively influenced by the use of a smart agent. The presence of a character makes it more likely that the user will feel anxiety and may therefore perform less well (Rickenberg \& Reeves, 2000). Software agents with a humanoid appearance have already been successfully tested in practice, for example the agents "WhizLow" and "Steve", which can guide learners through a virtual ship and computer environment.

\section{RESEARCH OBJECTIVE}

The research question deals with the integration of a smart agent in a virtual environment and its mode of interaction and visual appearance. The goal of this work consists in a first step of the development of an extensive literature research on the use of smart agents in Virtual Reality (VR). This should lead to insights into impacts and outcomes on the one hand, and influencing factors for successful use on the other.

\footnotetext{
${ }^{1}$ See http:/neuelehrkonzepte.ch
} 
In a second step, based on the knowledge gained from the first step, an own application in Unity will be designed and implemented. This immersive learning environment for mathematics has the sole purpose of identifying the preferred visualization of the target group's smart agent.

The following questions are addressed in detail:

1) How should a smart agent be integrated into virtual learning environments?

a. What are the requirements for the smart agent?

b. What are the requirements for the virtual environment?

c. Which scenarios can be realized with a smart agent?

d. Which potentials and problems arise when integrating a smart agent?

2) How should the interaction and the appearance of the smart agent be designed?

a. What are the tasks of a smart agent?

b. How do you communicate with a smart agent?

c. At what solution level does the smart agent operate?

d. What is the appearance of a smart agent? (Form, gestures, facial expressions)

The following chapters of this work are structured accordingly.

\section{INTEGRATION OF SMART AGENTS IN VIRTUAL WORLDS}

Virtual learning environments often lack interactive pedagogical support to encourage learning activities and motivate learners. smart agents should help to fill these gaps. It is therefore necessary to equip the agents with the right skills (Soliman M. \& Guetl, 2010).

According to (Bendel, 2003), smart agents in a virtual environment must perform tasks on behalf of people or perform actions to support or relieve users in their work. In order for smart agents to perform this support function, it is necessary to clarify what requirements are placed on both the smart agents and the virtual environment in which they interact with humans. Depending on the requirements, possible areas of application or scenarios can be developed in which smart agents can be used or where their use is not feasible.

Agents can be used to represent objects or even human-like beings. For example, a simulated aircraft can be just as much a virtual agent as a simulated enemy fighter. Virtual agents make simulations dynamic and help to develop them in interesting and unexpected ways from the learner's point of view. These agents are also called "non-playing characters" (NPC) in game environments. These NPCs are also found in most 3D games. Agents can assume different roles in applications and are each equipped with different pedagogical skills (Rickel, 2001).

No user intervention is required to execute functions. The agents can retrieve the actions autonomously, according to the rules formulated in the software (Nikodemus, 2017). Agent behavior can range from simple script-driven actions to complex decision making in response to the simulation context (Ramachandran, D., \& Stottler, R. (2004)).

Because agents operate in environments that are characterized by certain dynamics, the agent must be able to perceive state changes in order to perform the correct action. This means that the agent must have the ability to constantly analyze knowledge of the environment via sensors. An intelligent agent possesses the above-mentioned characteristics, which therefore also stands for the proximity to the field of artificial intelligence (AI) (Nikodemus, 2017).

\subsection{Requirements for Smart Agents}

Based on (Bendel, 2003), (Bendel, O., Schnöring, K., \& Back, A. (2002)), (Brenner et al., 1998), (Soliman M. $\&$ Guetl, 2010), (Rickel, 2001) the following requirements for an intelligent pedagogical agent are relevant for the context here:

1) Multimodal conversation skills with learners

2) Embodiment and Animation

3) Understanding and influencing the environment, learning objects and the learner

4) pedagogical competences with functions for learners, such as intelligent support and guidance and awareness of the learning activity to enable expert reactions

5) Central point of interaction between the learner and the environment that supports the learner verbally and non-verbally. 


\subsection{Requirements for the Virtual Learning Environment}

The special feature of virtual realities is their interactivity. This leads to learners being able to move in the virtual environment in a self-controlled manner and thus change their point of view and perspective, for example. In addition, learners can perform actions towards objects and persons who are in the virtual world (ITWissen.info, 2015).

An important prerequisite for the successful creation of a virtual learning environment is the examination of the learning object. According to (ITWissen.info, 2015) a distinction can be made between exploration worlds, training worlds and construction worlds.

For the present work, the development of a training world as a virtual learning space is used. The learners are given mathematical tasks which they have to solve with the help of the smart agent. The environment itself is not the focal point when solving the task, but rather provides the framework. Therefore, the learning environment should be designed as unobtrusive as possible, i.e. without distracting elements.

\subsection{Possible Scenarios}

In contrast to reality, simulated worlds can make specific things visible and thus understandable. This also makes it possible to train very unlikely scenarios. This results in a benefit from the simulation itself that can exceed that of classical learning in reality. Another advantage is the cost efficiency of simulations. In some cases, the necessary acquisition costs can be amortized after only two to three years. Further advantages of the use of simulations are also: harmlessness, mobility, no material wear, no damage to expensive equipment, practical, realistic training situation and modification of environmental variables (e.g., weather, light conditions, error messages from equipment) (Höntzsch et al., 2013).

If one restricts oneself to school-based applications, the following advantages may arise (Youngblut, 1998):

1) Visualization of abstract concepts

2) Observation of events at atomic or planetary level

3) Visits to distant geographical regions

4) Interaction with events that are not available due to distance, time or safety factors

5) Individualization specifically also through the use of smart agents

The present study focuses on the latter point. With the smart agent, learners can solve tasks according to their learning level and pace.

\subsection{Potentials and Challenges}

Based on (Bittner et al., 2019), (Bendel, O., Schnöring, K., \& Back , A. (2002)), (ITWissen.info, 2015), (Soliman M. \& Guetl, 2013) the following relevant points arise for this work:

1) Getting used to the smart agent: the first contact with a smart agent is unfamiliar and it takes some getting used to in order to be able to interact with it in a goal-oriented way.

2) Intelligence of the smart agent: an attempt is made to record all communication history in order to enable comprehensive and error-free communication.

3) Over-expectations of the smart agent: before the start of the learning unit, the smart agent should inform the learner about its functional scope.

4) Technical immaturity of the smart agent: in order to avoid possible problems associated with technical immaturity, the smart agent should, as stated in the previous point, point out its functional scope and explain where its limits lie. Particularly in communication, care should be taken to ensure that the smart agent provides short and concise inputs, which are also displayed textually where appropriate.

\section{INTERACTION AND VISUALIZATION}

The use of intelligent agents can increase the user's commitment to the virtual learning experience (Lester et al., 1997), (Van Mulken et al., 1998). They also promote motivation and self-confidence of the learners and can prevent negative affective states and minimize unwanted associations (e.g. frustration, boredom or fear of failure). It has also been shown that the use of several virtual characters with different but complementary roles 
can have further positive effects (Wißner et al., 2011). Kim and Baylor reported in their work on virtual learning guides on the three different qualities of competence, activity and realism (A. L. Baylor \& Kim, 2004), (Y. Kim et al., 2006). The competence assigned to a virtual character depends on the role the character takes on. For example, a teacher-like expertise can weaken motivational aspects. An intelligent agent with high competence can achieve good learning effects, whereas low competence leads to an increase in the learner's self-esteem, self-confidence and sense of responsibility. This is also useful when beginners are to be introduced to learning. The imitation of human behavior of the virtual character helps additionally. However, too realistic a design can lead to unrealistic expectations, disappointments and irritation. The classical role of an intelligent agent is that of a teacher (Wißner et al., 2011).

\subsection{The Tasks of a Smart Agent}

In a work by (Jawahar, 2015) a number of different agent descriptions were investigated. Agents are often characterized by the following attributes: autonomous, goal-oriented, cooperative, flexible, self-starting and adaptive.

Results from PACT (Pittsburgh Advanced Cognitive Tutor) Geometry Tutor indicate that learners lack the metacognitive skills and mathematical reading skills necessary to take full advantage of the tutor's on-request help or online glossary. A tutor should provide more support. The decision when learners can benefit from the tutor's online help should not be left to the learners alone. Rather, the tutor should help them to develop metacognitive skills. Using a model of metacognitive strategies, the tutor could help learners to use the glossary effectively.

The following three tasks appear to be useful:

1) The intelligent agent must act as a tutor (mentor), so that he not only gives the answer, but also the context for it, as well as further information.

2) The intelligent agent must be present for the learners in every phase.

3) The smart agent must become active on its own if the learners are not making any progress. This can be defined by a predefined behavior catalog.

\subsection{Interaction}

Agents can be text-based or language-based. For example, the agent can communicate by voice, but the response can then be via text input or a selection menu. The literature reveals that the voice channel is the most successful for both reception and transmission (Fleischer et al., 2008). Furthermore, it can be stated that the results of verbal instructions are better in every case, regardless of whether they were given purely verbally or with the support of a virtual agent.

Therefore, for the use of a smart agent as a tutor, it can be stated that the interaction should be carried out via verbal instructions from the agent and verbal feedback from the user, whereby the statements of the smart agent can also be presented in text form.

\subsection{Hints from the Smart Agent}

In the study by (Aleven \& Koedinger, 2000) which was conducted on 15-year-old learners, showed that intelligent help systems of a tutor are used more often than a mere glossary function. However, young people often wait too long before they ask for a clue. In addition, they did not seem to benefit much from the medium indication levels. In most cases where they asked for help, the learners repeated their requests for help until they received the solution.

Based on this insight, three levels of help are offered, but not all of them have to be passed through. It depends on the smart agent's assessment of which layer to use. This is defined based on a rule set.

1. Task assistance:

The task is explained by the smart agent and it is explained what exactly has to be done.

2. Solution hint:

A solution hint is given on how the learners can come to the solution and what is expected of them.

3. Solution:

It is specified how the problem can be solved and how to proceed (exact solution with explanation of the smart agent). 


\subsection{Visualization}

When designing a virtual agent, the visual presence and appearance of the agent is of high importance. These factors in particular can have a positive influence on the motivation of learners, regardless of the underlying technical complexity. This means that one voice alone (whether human or machine generated) with the same convincing message is not enough (A. L. Baylor, 2009). For example, Baylor \& Ryu found that providing an anthropomorphic agent (either static or animated) together with a human voice led to a greater perception of the credibility of the agent in the context of a learning environment (A. Baylor \& Ryu, 2003).

Further research by (Rosenberg-Kima et al., 2007, 2007) also shows that the visual presence of the agent has a large influence on the perceived benefit of the topic, a large influence on self-efficacy in success and a moderate effect on interest in the topic.

Another insight is that pedagogical actors should have human-like personalities in order to create a social context for natural learning (A. L. Baylor \& Kim, 2005), (Van Mulken et al., 1998).

In a contribution by (K. Kim et al., 2018), it was investigated how visual executions and social aspects of behavior influence the perception of an agent. It is assumed that a user's confidence in an agent's ability to perform tasks is improved if the agent is supplemented with a human body and social behaviors.

The visualization should therefore be done in such a way that it enables speech, gestures and movement in space. A human visualization seems to be preferred. However, this can also be done with animals with human characteristics. Apparently there is no uniform knowledge on the question of the optimal appearance in a young target audience. This question will be investigated in the following chapter by means of a field experiment.

\section{FIELD EXPERIMENT}

In order to test the visualization in an environment familiar to the learners, a virtual classroom was ${ }^{2}$ developed in which mathematical calculation tasks have to be solved on a blackboard. For the four visualizations (seeFigure 1), avatars from the Unity Asset Store were used. To make the avatars look realistic, they were equipped with simple animation effects (e.g., idle behavior). The avatars guide the learners through the scenario with the different tasks by means of audio-visual voice output.

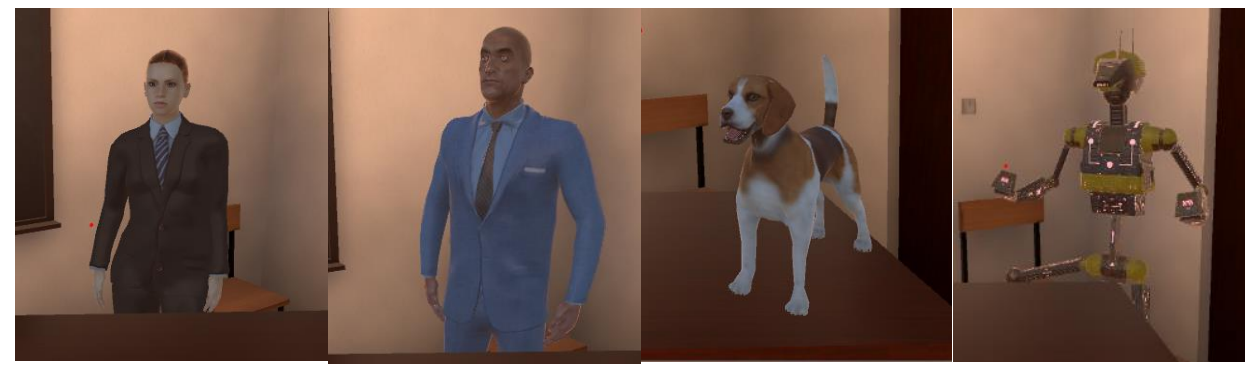

Figure 1. Visualizations for the smart agent

The scenario starts with a short explanation of the handling of the VR environment as well as the use of the VR controllers and then starts with the solution of the four mathematical tasks, each of which is distinguished by different visualizations of the smart agent (seeTable 1).

Table 1. Mathematical Tasks and the respective visualization of the smart agent and voice

\begin{tabular}{|l|l|l|}
\hline Mathematical task & Visualization & Voice \\
\hline Form the prime factorization of the number 100 & Female humanoid & de-DE, KatjaNeural \\
\hline $90: \mathrm{x}=-45$ & Male humanoid & de-CH, Karsten \\
\hline$-15: 3-(-1)=?$ & Animal & de-DE, Hedda \\
\hline Find $\mathrm{x} ; 19-2 \mathrm{x}=33$ & Robot & de-DE, Stefan, Apollo \\
\hline
\end{tabular}

\footnotetext{
${ }^{2}$ The application has been tested with HTC Vive and is available at

https://zhaw-my.sharepoint.com/:f:/g/personal/kell_zhaw_ch/EgkkMcPOv7ZHuvv0UrtRy7MBzNvzpb-v1NyAI8o2aB9tAA?e=uJhTh0
} 


\subsection{Participants}

A total of 15 students of a secondary school ( 8 girls and 7 boys) between 12 and 14 years participated in the field experiment and the subsequent survey. As mentioned above, 30 minutes were calculated for each test person (15 minutes testing of the VR application including observation and 15 minutes questioning about the application)

\subsection{Questionnaire}

The questionnaire used ${ }^{3}$ to evaluate the agent's design is based on the User Experience Model by (Mahlke, 2002). Mahlke assumes that information is processed on different experience dimensions when interacting with an application.

The following four aspects were integrated as experience dimensions: perceived benefit, user-friendliness, perceived hedonic quality and visual attractiveness. Perceived utility and usability are defined in Davis' Technology Acceptance Model (TAM) (Davis, 1989) and represent instrumental quality aspects. The concept of hedonic quality and that of visual attractiveness are examined as non-instrumental quality aspects. (Mahlke, 2005)

The questionnaire used consists of eight different questions (Table 2). For each question, learners have five answer categories at their disposal: (1) I totally agree, (2) I mostly agree, (3) I mostly disagree, (4) I totally disagree and (5) I don't know. In addition to the ten closed questions, the learners were also asked two open questions. This gave the opportunity to make general comments on the tested VR unit.

\subsection{Results}

Before testing the VR application, all learners received a short instruction on how to use the hardware and some inputs for the subsequent test in VR. Although the learners were instructed in advance and during the application to always direct their questions to the smart agent, a strikingly large number of test subjects turned to the project group when they were at a loss with a task. However, since the smart agent also answered these questions each time while the members of the project group replied, the smart agent's statements could hardly be understood. The fact that the smart agent is located in a corner of the classroom behind a lectern, but the learner is frontally in front of a blackboard and thus hardly notices the agent, favoured this behaviour.

The four tasks chosen for the test procedure were all from the subject of mathematics and were selected according to the level. However, for the learners to interact with the smart agent, the tasks had to be sufficiently difficult. Otherwise, the agent's assignment would have been invalidated. The test itself showed that the chosen tasks were challenging for all learners and that they were dependent on the help of the smart agent. Despite his advice, many learners were unable to solve the task at all and were then moved on to the next task as time ran out. It is assumed that this completely new way of learning and operating the hardware simply required too much attention for some learners and that they could not concentrate on the actual task.

Table 2. Results of survey

\begin{tabular}{|l|l|l|l|l|l|}
\hline Question & N & Min & Max & Average & Deviation \\
\hline Age & 60 & 12 & 14 & 12.7 & 0.5 \\
\hline The statements of the agent helped me to solve the tasks. & 37 & 1 & 3 & 2.62 & 0.7 \\
\hline The agent helped me to solve the tasks faster and easier. & 31 & 1 & 4 & 2.52 & 1 \\
\hline $\begin{array}{l}\text { The agent and his statements were clear and } \\
\text { understandable. }\end{array}$ & 48 & 1 & 4 & 2.83 & 1.1 \\
\hline The interaction with the agent was easy. & 37 & 1 & 4 & 2.84 & 0.6 \\
\hline The agent seemed trustworthy. & 38 & 1 & 4 & 3.24 & 0.9 \\
\hline The agent was appropriate for the environment. & 50 & 1 & 4 & 3.2 & 0.8 \\
\hline The agent was appealing. & 48 & 1 & 4 & 3.27 & 0.7 \\
\hline $\begin{array}{l}\text { I could imagine using the agent in a virtual learning } \\
\text { room. }\end{array}$ & 42 & 1 & 4 & 3.14 & 1 \\
\hline
\end{tabular}

1: I totally agree; 2: I mostly agree; 3: I mostly disagree; 4: I totally disagree

\footnotetext{
${ }^{3}$ The questionnaire is available for download at https://drive.switch.ch/index.php/s/xv87sbXpEp2uq8p/download
} 


\section{CONCLUSION}

As can be seen from the theoretical foundations of this work, various aspects must be considered when developing a smart agent. Thus, it must be clear which goals are to be achieved with the integration of a virtual agent and what role it plays in this process. Since the smart agent should constantly interact with the user, it must be able to simulate a behaviour that is as similar to human beings as possible (e.g., communication, facial expressions/gestures) and adequately sophisticated, whereby even small errors in the conception can have a negative influence on the user experience. The interaction with smart agents takes getting used to, especially considering the age of the users. It therefore takes time to adapt to this form of communication and learning.

The conducted field experiment can only give hints about the preferred visualization and interaction. The number of test persons is too small. In addition, for the learners, the handling of the hardware as well as the first-time experience with an immersive virtual learning environment suggests that the results may be biased and cannot be generalised. Nevertheless, it is interesting that a female, humanoid visualization of the smart agent seems to be the most appropriate. Similarly, a smart agent seems to be acceptable for assistance in solving tasks. The results of the survey are probably not convincing, but a possible optimization of the behavior gives hope for a future improvement.

\section{ACKNOWLEDGEMENT}

This work was made possible by four students of the MSc Business Informatics course.

\section{REFERENCES}

Aleven, V., \& Koedinger, K. R. (2000). Limitations of student control: Do students know when they need help? International Conference on Intelligent Tutoring Systems, 292-303.

Baylor, A. L. (2009). Promoting motivation with virtual agents and avatars: Role of visual presence and appearance. Philosophical Transactions of the Royal Society B: Biological Sciences, 364(1535), 3559-3565.

Baylor, A. L. (2011). The design of motivational agents and avatars. Educational Technology Research and Development, 59(2), 291-300.

Baylor, A. L., \& Kim, Y. (2005). Simulating instructional roles through pedagogical agents. International Journal of Artificial Intelligence in Education, 15(2), 95-115.

Baylor, A. L., \& Kim, Y. (2004). Pedagogical agent design: The impact of agent realism, gender, ethnicity, and instructional role. International Conference on Intelligent Tutoring Systems, 592-603.

Baylor, A., \& Ryu, J. (2003). The API (Agent Persona Instrument) for assessing pedagogical agent persona. EdMedia+ Innovate Learning, 448-451.

Bendel, O. (2003). Pädagogische Agenten im Corporate E-Learning. Dissertation [PhD Thesis]. Universität St. Gallen.

Bendel, O., Schnöring, K., \& Back, A. (2002). Potenziale pädagogischer Agenten. St. Gallen: Universität St. GallenGoogle-Suche. (n.d.). Retrieved January 23, 2020, from https://www.google.com/search?client=firefox-be\&q=Bendel $\% 2 \mathrm{C}+0 . \% 2 \mathrm{C}+\mathrm{Schn} \% \mathrm{C} 3 \% \mathrm{~B} 6$ ring $\% 2 \mathrm{C}+\mathrm{K} . \% 2 \mathrm{C}+\% 26+\mathrm{Back}+\% 2 \mathrm{C}+\mathrm{A} .+\% 282002 \% 29 .+$ Potenziale $+\mathrm{p} \%$ $\mathrm{C} 3 \%$ A4dagogischer+Agenten.+St.+Gallen\%3A+Universit\%C3\%A4t+St.+Gallen

Bittner, E. A., Oeste-Reiß, S., Ebel, P. A., \& Söllner, M. (2019). Mensch-Maschine-Kollaboration: Grundlagen, Gestaltungsherausforderungen und Potenziale für verschiedene Anwendungsdomänen. HMD Praxis Der Wirtschaftsinformatik, 56(1), 34-49.

Brenner, W., Zarnekow, R., \& Wittig, H. (1998). Intelligente Softwareagenten: Grundlagen und Anwendungen. Springer-Verlag. https://www.springer.com/de/book/9783662121047

Davis, F. D. (1989). Perceived usefulness, perceived ease of use, and user acceptance of information technology. MIS Quarterly, 319-340.

Fasihfar, Z., \& Rokhsati, H. (2017). Study of application of intelligent agents in e-learning systems. Bulletin de La Société Royale Des Sciences de Liège, 86, 398-405.

Fleischer, I., Plozer, C., Sträfling, N., \& Witschel, T. (2008). Der Einfluss des Erscheinungsbildes pädagogischer Agenten in computerbasierten Lernprogrammen auf die Motivation des Lerners und den Lernerfolg. Forschungsbericht vorgelegt im Fachgebiet Sozialpszchologie. 
Höntzsch, S., Katzky, U., Bredl, K., Kappe, F., \& Krause, D. (n.d.). Simulationen und simulierte Welten. Lernen in immersiven Lernumgebungen. 10.

ITWissen.info. (2015, February 3). Virtuelle Realität. http://www.itwissen.info/Virtuelle-Realitaet-virtual-reality-VR.html

Jawahar, J. (2015). A study on role of intelligent agent in education. International Journal of Applied Engineering Research, 10(76), 497-501.

Keller, T., Glauser, P., Ebert, N., \& Brucker-Kley, E. (2018). VIRTUAL REALITY AT SECONDARY SCHOOL-FIRST RESULTS. Proceedings of the 15th International Conference on Cognition and Exploratory Learning in the Digital Age (CELDA 2018), 53-60.

Keller, T., Hagen, F., \& Brucker-Kley, E. (2019). A field study about the impact of a VR learning unit. CELDA 2019 16th International Conference Cognition and Exploratory Learning in Digital Age, Cagliari, Italy, 7-9 November 2019, 307-314.

Keller, T., Hebeisen, A., \& Brucker-Kley, E. (2018). Integration of children with special needs in mathematics through virtual reality. Proceedings of the 15th International Conference on Cognition and Exploratory Learning in the Digital Age (CELDA 2018), 30-37.

Kim, K., Boelling, L., Haesler, S., Bailenson, J., Bruder, G., \& Welch, G. F. (2018). Does a digital assistant need a body? The influence of visual embodiment and social behavior on the perception of intelligent virtual agents in AR. 2018 IEEE International Symposium on Mixed and Augmented Reality (ISMAR), 105-114.

Kim, Y., \& Baylor, A. L. (2016). based design of pedagogical agent roles: A review, progress, and recommendations. International Journal of Artificial Intelligence in Education, 26(1), 160-169.

Kim, Y., Baylor, A. L., \& Group, P. (2006). Pedagogical agents as learning companions: The role of agent competency and type of interaction. Educational Technology Research and Development, 54(3), 223-243.

Lester, J. C., Converse, S. A., Kahler, S. E., Barlow, S. T., Stone, B. A., \& Bhogal, R. S. (1997). The persona effect: Affective impact of animated pedagogical agents. Proceedings of the ACM SIGCHI Conference on Human Factors in Computing Systems, 359-366. https://doi.org/10.1145/258549.258797

Mahlke, S. (2005). An integrative model on web user experience. Proceeding of ICWI2005, 2, 91-95.

Mahlke, S. (2002). Factors influencing the experience of website usage. CHI'02 Extended Abstracts on Human Factors in Computing Systems, 846-847.

Nikodemus, P. (2017). Lernprozessorientiertes Wissensmanagement und Kooperatives Lernen. Springer.

Ramachandran, D., \& Stottler, R. (2004). The Many Roles of Virtual Agents in Training. San Mateo, CA: Stottler Henke Associates, Inc-Google-Suche. (n.d.). Retrieved January 23, 2020, from https://www.google.com/search?client=firefox-be\&q=Ramachandran,+D.,+\%26+Stottler,+R.+(2004).+The+Many+Roles+of+Virtual+Agents+in+Training.+San+Ma teo,+CA:+Stottler+Henke+Associates,+Inc\&spell=1\&sa=X\&ved=2ahUKEwj488aH9pnnAhXI4KQKHXR1DYQQB SgAegQIBhAq\&biw=1498\&bih=798

Rickel, J. (2001). Intelligent virtual agents for education and training: Opportunities and challenges. International Workshop on Intelligent Virtual Agents, 15-22.

Rickenberg, R., \& Reeves, B. (2000). The effects of animated characters on anxiety, task performance, and evaluations of user interfaces. Proceedings of the SIGCHI Conference on Human Factors in Computing Systems, 49-56.

Rosenberg-Kima, R. B., Baylor, A. L., Plant, E. A., \& Doerr, C. E. (2007). The importance of interface agent visual presence: Voice alone is less effective in impacting young women's attitudes toward engineering. International Conference on Persuasive Technology, 214-222.

Soliman, M., \& Guetl, C. (2013). Implementing Intelligent Pedagogical Agents in virtual worlds: Tutoring natural science experiments in OpenWonderland. 2013 IEEE Global Engineering Education Conference (EDUCON), 782-789. https://doi.org/10.1109/EduCon.2013.6530196

Soliman, M., \& Guetl, C. (2010). Intelligent pedagogical agents in immersive virtual learning environments: A review. The $33 r d$ International Convention MIPRO, 827-832.

Van Mulken, S., André, E., \& Müller, J. (1998). The persona effect: How substantial is it? In People and computers XIII (pp. 53-66). Springer.

Wißner, M., Beek, W., Lozano, E., Mehlmann, G., Linnebank, F., Liem, J., Häring, M., Bühling, R., Gracia, J., \& Bredeweg, B. (2011). Increasing Learners' Motivation through Pedagogical Agents: The Cast of Virtual Characters in the DynaLearn ILE. International Workshop on Agents for Educational Games and Simulations, 151-165.

Youngblut, C. (1998). Educational uses of virtual reality technology. Institute for Defense Analyses Alexandria VA. 